\title{
ON THE MULTIPLICITY OF THE EIGENVALUES OF THE VECTORIAL STURM-LIOUVILLE EQUATION
}

\author{
CHIEN-WEN LIN
}

\begin{abstract}
Let $Q(x)$ be a continuous $m \times m$ real symmetric matrix-valued function defined on $[0,1]$, and denote the Sturm-Liouville operator $-\frac{d^{2}}{d x^{2}}+Q(x)$ as $L_{Q}$ with $Q(x)$ as its potential function. In this paper we prove that for each Dirichlet eigenvalue $\lambda_{*}$ of $L_{Q}$, the geometric multiplicity of $\lambda_{*}$ is equal to its algebraic multiplicity. Applying this result, we get a necessary and sufficiently condition such that each Dirichlet eigenvalue of $L_{Q}$ is of multiplicity $m$.
\end{abstract}

\section{Introduction}

In this paper we shall study some problems related to the multiplicity of the eigenvalue of the following vectorial Sturm-Liouville equation:

$$
\left\{\begin{array}{l}
y^{\prime \prime}(x)+\left(\lambda I_{m}-Q(x)\right) y(x)=0, \\
y(0)=y(1)=0
\end{array}\right.
$$

where $I_{m}$ is the identity operator on $\mathbb{R}^{m}, Q(x)$ is an $m \times m$ real symmetric matrix-valued continuous function, and $y(x)$ is an $\mathbb{R}^{m}$-valued function. Denote $L_{Q}=-\frac{d^{2}}{d x^{2}}+Q(x)$ and call it the Sturm-Liouville operator with the potential function $Q(x)$. We say that a number $\lambda_{*}$ is a Dirichlet eigenvalue of $L_{Q}$ if and only if the equation (1) has a nontrivial solution. Such a solution is called a Dirichlet eigenfunction of $L_{Q}$ correponding to the eigenvalue $\lambda_{*}$. Let $m_{g}\left(\lambda_{*}\right)$ denote the geometric multiplicity of the eigenvalue $\lambda_{*}$, which is the dimension of the subspace of eigenfunctions corresponding to $\lambda_{*}$. The collection of all Dirichlet eigenvalues of $L_{Q}$ is called the Dirichlet spectrum of $L_{Q}$, and denoted by $\sigma_{D}\left(L_{Q}\right)$. According to the selfadjointness of $Q(x)$, we know that all Dirichlet eigenvalues are real. Counting the geometric multiplicity of the eigenvalues, we arrange the Dirichlet eigenvalues of $L_{Q}$ in ascending order as:

$$
\lambda_{1} \leqslant \lambda_{2} \leqslant \lambda_{3} \leqslant \cdots \leqslant \lambda_{k} \leqslant \cdots .
$$

This research was supported in part by the National Science Council of the Republic of China, Taiwan. 
In order to study the Sturm-Liouville eigenvalue problem, we introduce the following initial value problem:

$$
\left\{\begin{array}{l}
Y^{\prime \prime}(x)+\left(\lambda I_{m}-Q(x)\right) Y(x)=0_{m} \\
Y(0)=0_{m}, \quad Y^{\prime}(0)=I_{m}
\end{array}\right.
$$

and denote its solution by $Y(x ; \lambda)$. Then $\lambda_{*}$ is a Dirichlet eigenvalue of $L_{Q}$ if and only if $\operatorname{det} Y\left(1 ; \lambda_{*}\right)=0$, and $m_{g}\left(\lambda_{*}\right)=\operatorname{dim}\left(\operatorname{ker} Y\left(1 ; \lambda_{*}\right)\right)$. Let $m_{a}\left(\lambda_{*}\right)$ denote the algebraic multiplicity of the eigenvalue $\lambda_{*}$, which is determined by the following equality:

$$
m_{a}\left(\lambda_{*}\right)=\max \left\{n \in \mathbb{Z}^{+} \mid\left(\lambda-\lambda_{*}\right)^{-n} \operatorname{det} Y(1 ; \lambda) \text { is analytic at } \lambda_{*}\right\} .
$$

It is known that for each $\lambda_{*} \in \sigma_{D}\left(L_{Q}\right), m_{g}\left(\lambda_{*}\right) \leqslant m_{a}\left(\lambda_{*}\right)$. With the help of a homotopy method motivated by the approach of L. Bers in his paper [2], we prove the following theorem:

Theorem 2. Suppose that $Q(x) \in C\left([0,1] ; \mathscr{L}\left(\mathbb{R}^{m}\right)\right)$, and $Q(x)=Q(x)^{*}$ for all $x \in[0,1]$. Then $m_{a}\left(\lambda_{*}\right)=m_{g}\left(\lambda_{*}\right)$ for any $\lambda_{*} \in \sigma_{D}\left(L_{Q}\right)$.

On the other hand, for the Dirichlet eigenvalues of $L_{Q}$, it is known that $m_{g}\left(\lambda_{*}\right) \leqslant m$ for each $\lambda_{*} \in \sigma_{D}\left(L_{Q}\right)$. In the works of C-L. Shen $[7,8]$ about the inverse eigenvalue problems related to equation (1), it was shown that if $Q(x)$ is an even function, and $m_{g}\left(\lambda_{*}\right)=m$ for each $\lambda_{*} \in \sigma_{D}\left(L_{Q}\right)$, then $Q(x)$ is a diagonal matrix-valued function. Note that $m_{g}\left(\lambda_{*}\right)=m$ if and only if $Y\left(1 ; \lambda_{*}\right)=0_{m}$. Therefore it is interesting to find a spectral condition, only depending on the eigenvalues of equation (1), to tell whether all Dirichlet eigenvalues of $L_{Q}$ are of multiplicity $m$. We use a homotopy method to study this problem. Denote $Y(x ; \lambda ; t)$ as the solution of the following initial value problem:

$$
Y^{\prime \prime}(x)+(\lambda I-t Q(x)) Y(x)=0, \quad Y(0)=0, \quad Y^{\prime}(0)=I,
$$

where $t \in[0,1]$. Then for sufficiently large $l \in \mathbb{N}$ we know that $Y\left(1 ;\left(l+\frac{1}{2}\right)^{2} \pi^{2} ; t\right)$ is an invertible matrix for all $t \in[0,1]$. Thus the following contour integral makes sense:

$$
M_{l}(t)=\frac{1}{2 \pi i} \oint_{|\lambda|=\left(l+\frac{1}{2}\right)^{2} \pi^{2}} \frac{\frac{\partial}{\partial \lambda} \operatorname{det} Y(1 ; \lambda ; t)}{\operatorname{det} Y(1 ; \lambda ; t)} d \lambda .
$$

Since $M_{l}(t)$ is a continuous positive integer-valued function on $[0,1]$, then $M_{l}(1)=M_{l}(0)=$ $l m$. Thus there are $l m$ Dirichlet eigenvalues (counting multiplicity) smaller than $\left(l+\frac{1}{2}\right)^{2} \pi^{2}$. According to these argument, we obtain a necessary and sufficiently condition which implies all Dirichlet eigenvalues of $L_{Q}$ are of multiplicity $m$.

Theorem 4. Suppose that $Q(x) \in C\left([0,1] ; \mathscr{L}\left(\mathbb{R}^{m}\right)\right)$, and $Q(x)=Q(x)^{*}$ for all $x \in[0,1]$. Then all Dirichlet eigenvalues of $L_{Q}$ are of multiplicity $m$ if and only if

$$
\sigma_{D}\left(L_{Q}\right)=\left\{n^{2} \pi^{2}+\tau_{n} \mid\left\{\tau_{n}\right\}_{n=1}^{\infty} \text { is a bounded sequence }\right\} .
$$


Furthermore, $\left\{\tau_{n}\right\}_{n=1}^{\infty}$ is a convergent sequence, and

$$
\int_{0}^{1} Q(t) d t=\left\{\lim _{n \rightarrow \infty} \tau_{n}\right\} I_{m}
$$

\section{Preliminary}

In order to study the Sturm-Liouville eigenvalue problem with the selfadjoint $m \times m$ matrix-valued potential $Q(x)$, we consider the following initial value problems:

$$
\left\{\begin{array}{l}
Y^{\prime \prime}(x ; \lambda)+\left(\lambda I_{m}-Q(x)\right) Y(x ; \lambda)=0_{m} \\
Y(0 ; \lambda)=0_{m}, \quad Y^{\prime}(0 ; \lambda)=I_{m}
\end{array}\right.
$$

where $0_{m}$ is the $m \times m$ zero matrix. We have that

$$
Y(x ; \lambda)=\frac{\sin \sqrt{\lambda} x}{\sqrt{\lambda}} I_{m}+\int_{0}^{x} \frac{\sin \sqrt{\lambda}(x-t)}{\sqrt{\lambda}} Q(t) Y(t ; \lambda) d t,
$$

furthermore,

$$
Y(1 ; \lambda)=\frac{\sin \sqrt{\lambda}}{\sqrt{\lambda}} I_{m}-\frac{\cos \sqrt{\lambda}}{2 \lambda} \int_{0}^{1} Q(x) d t+O\left(\frac{\exp \left(\|Q\|_{\infty}|\Im \sqrt{\lambda}|\right)}{|\lambda|^{\frac{3}{2}}}\right),
$$

where $\|Q\|_{\infty}=\sup \left\{\|Q(x)\|_{L\left(\mathbb{R}^{m}\right)} \mid x \in[0 ; 1]\right\}$, and we have that as a function of $\lambda, Y(x ; \lambda)$ is a matrix-valued entire function. It is well known that $\lambda_{*} \in \sigma_{D}\left(L_{Q}\right)$, if and only if $Y\left(1 ; \lambda_{*}\right)$ has a nontrivial kernel, thus $\operatorname{det} Y\left(1 ; \lambda_{*}\right)=0$.

To analyze the distribution of the zeros of $\operatorname{det} Y(1 ; \lambda)$ is helpful for our study of the structure of $\sigma_{D}\left(L_{Q}\right)$. Now we introduce a Rouché's theorem for analytic matrix valued-functions (see [G2, Ch.XI Thm. 9.2]). Let $\Phi(\lambda)$ be an analytic matrix-valued function defined on an open connected region $\Omega \subset \mathbb{C}$. Define

$$
\begin{aligned}
& m_{g}\left(\lambda_{0} ; \Phi\right)=\operatorname{dim} \operatorname{ker} \Phi\left(\lambda_{0}\right), \\
& m_{a}\left(\lambda_{0} ; \Phi\right)=\max \left\{n \in \mathbb{Z}^{+} \mid\left(\lambda-\lambda_{0}\right)^{-n} \operatorname{det} \Phi(\lambda) \text { is analytic at } \lambda_{0}\right\},
\end{aligned}
$$

where $m_{g}\left(\lambda_{0} ; \Phi\right)$ and $m_{a}\left(\lambda_{0} ; \Phi\right)$ are geometric and algebraic multiplicity of $\lambda_{0}$ corresponding to $\Phi(\lambda)$, respectively. Let $\Gamma$ be a Cauchy contour in $\Omega$ with inner domain $\Delta \subset \Omega$. We say that $\Phi(\lambda)$ is normal with respect to $\Gamma$ if $\Phi(\lambda)$ is invertible for all $\lambda \in \Gamma$. Applying the analyticity of $\Phi(\lambda)$, we have that $\operatorname{det} \Phi(\lambda)$ is also analytic on $\Omega$, thus there are only finitely many $\lambda_{*} \in \Delta$, such that $\Phi\left(\lambda_{*}\right)$ is noninvertible. Then we may define the following quantities:

$$
m_{g}(\Gamma ; \Phi)=\sum_{\lambda \in \Delta} m_{g}(\lambda ; \Phi), \quad m_{a}(\Gamma ; \Phi)=\sum_{\lambda \in \Delta} m_{a}(\lambda ; \Phi) .
$$

If $\Phi\left(\lambda_{*}\right)$ is selfadjoint, then we know $m_{a}\left(\lambda_{*} ; \Phi\right)=m_{g}\left(\lambda_{*} ; \Phi\right)$. But for nonselfadjoint $\Phi(\lambda)$ we only have that $m_{a}\left(\lambda_{*} ; \Phi\right) \geqslant m_{g}\left(\lambda_{*} ; \Phi\right)$. Gohberg and his coworkers proved the following Rouché's theorem for analytic matrix valued-functions (see [G2, Ch.XI Thm. 9.2]). 
Theorem 1. Let $\Phi(\lambda), \Psi(\lambda): \Omega \in \mathscr{C} \rightarrow \mathscr{L}\left(\mathbb{R}^{n}\right)$ be analytic matrix valued-functions, and assume that $\Phi$ is normal with respect to the Cauchy contour $\Gamma \in \Omega$. If

$$
\left\|\Phi(\lambda)^{-1} \Psi(\lambda)\right\|_{\mathscr{L}\left(\mathbb{R}^{n}\right)}<1, \quad \text { for all } \lambda \in \Gamma
$$

then the function $V(\lambda)=\Phi(\lambda)+\Psi(\lambda)$ is also normal with respect to $\Gamma$, and $m_{a}(\Gamma ; \Phi)=m_{a}(\Gamma ; V)$.

In the remaining part of this section we shall analyze the distribution of Dirichlet eigenvalues of $L_{Q}$. Applying the selfadjointness of $Q(x)$, we have that there exist an $m \times m$ unitary matrix $P$, such that

$$
P^{*}\left(\int_{0}^{1} Q(t) d t\right) P=\left(\begin{array}{cccc}
q_{1} & 0 & \cdots & 0 \\
0 & q_{2} & \cdots & 0 \\
\vdots & \vdots & \ddots & \vdots \\
0 & 0 & \cdots & q_{m}
\end{array}\right),
$$

where $\left\{q_{j}\right\}_{1}^{m}$ are eigenvalues of the constant matrix $\int_{0}^{1} Q(t) d t$, and $q_{1} \leqslant q_{2} \leqslant \cdots \leqslant q_{m}$. Applying (4), we have that

$$
\begin{aligned}
P^{*} Y(1 ; \lambda) P= & \frac{\sin \sqrt{\lambda}}{\sqrt{\lambda}} I_{m}-\frac{\cos \sqrt{\lambda}}{2 \lambda}\left(\begin{array}{cccc}
q_{1} & 0 & \cdots & 0 \\
0 & q_{2} & \cdots & 0 \\
\vdots & \vdots & \ddots & \vdots \\
0 & 0 & \cdots & q_{m}
\end{array}\right) \\
& +O\left(\frac{\exp \left(\|Q\|_{\infty}|\Im \sqrt{\lambda}|\right)}{|\lambda|^{\frac{3}{2}}}\right) .
\end{aligned}
$$

Denote

$$
\begin{aligned}
& \Phi(\lambda)=\frac{\sin \sqrt{\lambda}}{\sqrt{\lambda}} I_{m}-\frac{\cos \sqrt{\lambda}}{2 \lambda}\left(\begin{array}{cccc}
q_{1} & 0 & \cdots & 0 \\
0 & q_{2} & \cdots & 0 \\
\vdots & \vdots & \ddots & \vdots \\
0 & 0 & \cdots & q_{m}
\end{array}\right), \\
& \Psi(\lambda)=P Y(1 ; \lambda) P-\Phi(\lambda) .
\end{aligned}
$$

Since

$$
\operatorname{det} \Phi(\lambda)=\prod_{i=1}^{m}\left(\frac{\sin \sqrt{\lambda}}{\sqrt{\lambda}}-\frac{\cos \sqrt{\lambda}}{2 \lambda} q_{i}\right)
$$

Let $\mu_{l m+i}$ be the $(l m+i)$-th zero of $\operatorname{det} \Phi(\lambda)$. Applying the Rouché's theorem, we find that $\mu_{l m+i}$ satisfies

$$
\mu_{l m+i}-(l+1)^{2} \pi^{2}=q_{i}+O\left(\frac{1}{l}\right) .
$$


On the other hand, for sufficient large $l$, denote

$$
\gamma_{l}^{i}=\left\{\lambda \in \mathbb{C}|| \lambda-\mu_{l m+i} \mid=\varepsilon_{l}^{i^{i}},\right.
$$

where $\varepsilon_{l}^{i}$ is a suitable number, such that $\operatorname{det} \Phi(\lambda) \neq 0$ for all $\lambda$ inside $\gamma_{l}^{i}$ except $\mu_{l m+i}$, and

$$
\lim _{l \rightarrow \infty} \varepsilon_{l}^{i}=0,
$$

and $\left\|\Phi(\lambda)^{-1} \Psi(\lambda)\right\|_{\mathscr{L}\left(\mathbb{R}^{n}\right)}<1$ for any $\lambda \in \gamma_{l}^{i}$. According to Theorem 1, we know that

$$
m_{a}\left(\gamma_{l}^{i} ; \Phi\right)=m_{a}\left(\gamma_{l}^{i} ; \Phi+\Psi\right)=m_{a}\left(\gamma_{l}^{i} ; Y(1 ; \lambda)\right)=m_{a}\left(\mu_{l m+i} ; \Phi\right)
$$

This shows that the eigenvalues of the equation (1) appear near $(l+1)^{2} \pi^{2}+q_{i}$ for sufficient large $l$.

\section{On the analysis of the structure of $\sigma_{D}\left(L_{Q}\right)$}

The purpose in this section is to prove that $m_{a}\left(\lambda_{*} ; Y(1 ; \lambda)\right)=m_{g}\left(\lambda_{*} ; Y(1 ; \lambda)\right)$ for all $\lambda_{*} \epsilon$ $\sigma_{D}\left(L_{Q}\right)$, where $Q(x) \in \mathscr{L}\left(\mathbb{R}^{n}\right)$. Before we begin to prove our assertion, we need some notation. Consider the following auxiliary eigenvalue problem:

$$
\left\{\begin{array}{l}
y^{\prime \prime}(x)+\left(\lambda I_{m}-t Q(x)\right) y(x)=0 \\
y(0)=y(1)=0
\end{array}\right.
$$

and the following initial value problem:

$$
Y^{\prime \prime}(x)+\left(\lambda I_{m}-t Q(x)\right) Y(x)=0, \quad Y(0)=0, \quad Y^{\prime}(0)=I_{m},
$$

where $t \in[0,1]$. Denote $Y(x ; \lambda ; t)$ the solution of the equation (7), and let $\lambda_{n}^{g}(t)$ denote the $n$-th eigenvalue (counting geometric multiplicity) of (6). Then $\operatorname{det} Y\left(1 ; \lambda_{n}^{g}(t) ; t\right)=0$ for all $n \in \mathbb{N}$. Let $\lambda_{n}^{a}(t)$ be the $n$-th zero (counting algebraic multiplicity) of $\operatorname{det} Y(1 ; \lambda ; t)$. Then $\operatorname{ker} Y\left(1 ; \lambda_{n}^{a}(t)\right) \neq$ $\{0\}$. Thus for any $\lambda_{n}^{g}(t)$, there exists $k_{n}$, such that $\lambda_{k_{n}}^{a}(t)=\lambda_{n}^{g}(t)$, and for any $\lambda_{n}^{a}(t)$, there exists $j_{n}$, such that $\lambda_{j_{n}}^{g}(t)=\lambda_{n}^{a}(t)$.

Theorem 2. Suppose that $Q(x) \in C\left([0,1] ; \mathscr{L}\left(\mathbb{R}^{m}\right)\right)$, and $Q(x)=Q(x)^{*}$ for all $x \in[0,1]$. Then $m_{a}\left(\lambda_{*}\right)=m_{g}\left(\lambda_{*}\right)$ for any $\lambda_{*} \in \sigma_{D}\left(L_{Q}\right)$.

Proof. To prove our theorem, we have to prove that $m_{g}\left(\lambda_{*} ; Y(1 ; \lambda)\right)=m_{a}\left(\lambda_{*} ; Y(1 ; \lambda)\right)$, where $\lambda_{*} \in \sigma_{D}\left(L_{Q}\right)$. If we can prove that $\lambda_{n}^{g}(t)=\lambda_{n}^{a}(t)$ for all $t \in[0,1]$, and $n \in \mathbb{N}$, then $m_{g}\left(\lambda_{n}^{a}(t)\right.$; $Y(1 ; \lambda ; t))=m_{a}\left(\lambda_{n}^{g}(t) ; Y(1 ; \lambda ; t)\right)$. Since $Y(1 ; \lambda)=Y(1 ; \lambda ; 1)$, then our assertion holds. According to the definition of $\lambda_{n}^{a}(t), \lambda_{n}^{g}(t)$ and the continuity of $Y(1 ; \lambda ; t)$ corresponding to $t$-parameter, we know that $\left\{\lambda_{n}^{a}(t)\right\}_{n=1}^{\infty}$ and $\left\{\lambda_{n}^{g}(t)\right\}_{n=1}^{\infty}$ are two increasing sequences of continuous functions, and $\lambda_{n}^{a}(t) \leqslant \lambda_{n}^{g}(t)$ for all $t \in[0,1], n \in \mathbb{N}$. 
Suppose that there exists $j \in \mathbb{N}, t_{0} \in[0,1]$, such that $\lambda_{j}^{a}\left(t_{0}\right)<\lambda_{j}^{g}\left(t_{0}\right)$. Denote

$$
\mathscr{N}=\left\{t \in[0,1] \mid \text { There exist } j_{t} \text {, such that } \lambda_{j_{t}}^{a}(t)<\lambda_{j_{t}}^{g}(t)\right\}
$$

Since $t_{0} \in \mathscr{N}$, then $\mathscr{N}$ is a nonempty subset of $[0,1]$. On the other hand, for any $t_{*} \in \mathscr{N}$, according to the continuity of $\lambda_{j_{t_{*}}}^{a}(t)$ and $\lambda_{j_{*}}^{g}(t)$, there exists an open neighborhood $\mathscr{I}_{t_{*}}$ of $t_{*}$, such that for all $t \in \mathscr{I}_{t_{*}}$, we have that $\lambda_{j_{t_{*}}}^{a}(t)<\lambda_{j_{t_{*}}}^{g}(t)$. This implies that $\mathscr{I}_{t_{*}} \subset \mathscr{N}$, thus $\mathscr{N}$ is an relatively open subset of $[0,1]$. Furthermore, for any $t \in \mathscr{N}$, applying (5) we have that

$$
\limsup _{n \rightarrow \infty} \frac{\lambda_{n}^{g}(t)-\lambda_{n}^{a}(t)}{2 n \pi} \geqslant 1 .
$$

Let $t_{*}$ be an accumulation point of $\mathscr{N}$, but $t_{*} \notin \mathscr{N}$. Then there exist $\left\{t_{n}\right\}_{n=1}^{\infty} \subset \mathscr{N}$, such that $t_{n}$ converges to $t_{*}$ as $n$ tends to infinite. In the previous argument we know that

$$
\lambda_{l m+i}^{a}\left(t_{n}\right)-\lambda_{l m+i}^{a}\left(t_{*}\right)=\left(t_{n}-t_{*}\right) q_{i}+O\left(\frac{1}{l}\right)
$$

Denote $y_{n}(x ; t)$ the $n$-th eigenfunction of (6), such that $\int_{0}^{1}\left\|y_{n}(x ; t)\right\|^{2} d t=1$. Then applying the variational principle (see [4]), we have that

$$
\begin{aligned}
\lambda_{n}^{g}(t) \leqslant & \int_{0}^{1}\left\|y_{n}^{\prime}\left(x ; t_{*}\right)\right\|^{2} d x+\int_{0}^{1}\left\langle t Q(x) y_{n}\left(x ; t_{*}\right), y_{n}\left(x ; t_{*}\right)\right\rangle d t \\
= & \int_{0}^{1}\left\|y_{n}^{\prime}\left(x ; t_{*}\right)\right\|^{2} d x+\int_{0}^{1}\left\langle t_{*} Q(x) y_{n}\left(x ; t_{*}\right), y_{n}\left(x ; t_{*}\right)\right\rangle d t \\
& +\left(t-t_{*}\right) \int_{0}^{1}\left\langle Q(x) y_{n}\left(x ; t_{*}\right), y_{n}\left(x ; t_{*}\right)\right\rangle d t \\
= & \lambda_{n}^{g}\left(t_{*}\right)+\left(t-t_{*}\right) \int_{0}^{1}\left\langle Q(x) y_{n}\left(x ; t_{*}\right), y_{n}\left(x ; t_{*}\right)\right\rangle d t
\end{aligned}
$$

and

$$
\begin{aligned}
\lambda_{n}^{g}\left(t_{*}\right) \leqslant & \int_{0}^{1}\left\|y_{n}^{\prime}(x ; t)\right\|^{2} d x+\int_{0}^{1}\left\langle t_{*} Q(x) y_{n}(x ; t), y_{n}(x ; t)\right\rangle d t \\
= & \int_{0}^{1}\left\|y_{n}^{\prime}(x ; t)\right\|^{2} d x+\int_{0}^{1}\left\langle t Q(x) y_{n}(x ; t), y_{n}(x ; t)\right\rangle d t \\
& +\left(t_{*}-t\right) \int_{0}^{1}\left\langle Q(x) y_{n}(x ; t), y_{n}(x ; t)\right\rangle d t \\
= & \lambda_{n}^{g}(t)+\left(t_{*}-t\right) \int_{0}^{1}\left\langle Q(x) y_{n}(x ; t), y_{n}(x ; t)\right\rangle d t .
\end{aligned}
$$

Following the previous two inequalities and applying Hölder inequality, we find that

$$
\left|\lambda_{n}^{g}(t)-\lambda_{n}^{g}\left(t_{*}\right)\right| \leqslant\left|t-t_{*}\right| \cdot\|Q\|_{\infty} .
$$


Since $t_{*} \notin \mathscr{N}$, then $\lambda_{n}^{a}\left(t_{*}\right)=\lambda_{n}^{g}\left(t_{*}\right)$ for all $n \in \mathbb{N}$. According to (9) and (10), we have that

$$
\begin{aligned}
\lambda_{n}^{g}\left(t_{l}\right)-\lambda_{n}^{a}\left(t_{l}\right) & \leqslant\left|\lambda_{n}^{g}\left(t_{l}\right)-\lambda_{n}^{g}\left(t_{*}\right)\right|+\left|\lambda_{n}^{a}\left(t_{l}\right)-\lambda_{n}^{a}\left(t_{*}\right)\right| \\
& \leqslant\left|t_{l}-t_{*}\right|\left\{\max _{i=1, \cdots, m} q_{i}\right\}+O\left(\left|t_{l}-t_{*}\right|\right)+O\left(\frac{1}{\left[\frac{n}{m}\right]}\right) .
\end{aligned}
$$

This is a contradiction to the inequality (8). Thus $t_{*} \in \mathscr{N}$, and we find that $\mathscr{N}$ is a relatively closed subset of $[0,1]$. By the connectedness of $[0,1]$, and the assumption of $\mathscr{N}$ being nonempty, we conclude that $\mathscr{N}=[0,1]$. But $Y(1 ; \lambda ; 0)=\frac{\sin \sqrt{\lambda}}{\sqrt{\lambda}} I_{m}$, this shows that $\lambda_{n}^{a}(0)=\lambda_{n}^{g}(0)$ for all $n \in \mathbb{N}, 0 \notin \mathscr{N}$, which is absurd. Thus $\mathscr{N}$ is empty. Hence $\lambda_{n}^{g}(t)=\lambda_{n}^{a}(t)$ for all $n \in \mathbb{N}$.

Remark. If we consider the Neumann eigenvalue problem of $L_{Q}$ as follows:

$$
\left\{\begin{array}{l}
z^{\prime \prime}(x)+\left(\lambda I_{m}-Q(x)\right) z(x)=0, \\
z^{\prime}(0)=z^{\prime}(1)=0
\end{array}\right.
$$

and denote $Z(x ; \lambda)$ the solution of the following initial value problem:

$$
\left\{\begin{array}{l}
Z^{\prime \prime}(x ; \lambda)+\left(\lambda I_{m}-Q(x)\right) Z(x ; \lambda)=0_{m} \\
Z(0 ; \lambda)=I_{m}, \quad Z^{\prime}(0 ; \lambda)=0_{m}
\end{array}\right.
$$

Then we know that $\mu_{*}$ is in the Neumann spectrum $\sigma_{N}\left(L_{Q}\right)$ of $L_{Q}$ or a Neumann eigenvalue of $L_{Q}$, if and only if $\operatorname{det} Z^{\prime}\left(1 ; \mu_{*}\right)=0$. Applying the similar argument in the proof of Theorem 2 , we get that $m_{g}(\mu)=m_{a}(\mu)$ for all $\mu \in \sigma_{N}\left(L_{Q}\right)$. Thus for any Neumann eigenvalue of $L_{Q}$ its geometric multiplicity are equal to its algebraic multiplicity.

Denote $\gamma_{l}=\left\{\lambda \in \mathbb{C}:\left|\lambda-l^{2} \pi^{2}\right|=\left(l-\frac{1}{4}\right) \pi^{2}\right\}, l \in \mathbb{N}$. According to the identity (5), there exists a positive integer $M_{Q}$, such that

$$
\frac{1}{2 \pi i} \oint_{\gamma_{l}} \frac{\frac{\partial}{\partial \lambda} \operatorname{det} Y(1 ; \lambda)}{\operatorname{det} Y(1 ; \lambda)} d \lambda=m
$$

for any $l \geqslant M_{Q}, l \in \mathbb{N}$. Applying Theorem 2, we have that there are $m$ Dirichlet eigenvalues of $L_{Q}$ which are contained inside $\gamma_{l}$. On the other hand, according to (4), we have that

$$
Y\left(1 ;\left(l+\frac{1}{2}\right)^{2} \pi^{2} ; t\right)=\frac{(-1)^{l}}{\left(l+\frac{1}{2}\right) \pi} I_{m}+t O\left(\frac{1}{\left(l+\frac{1}{2}\right)^{3} \pi^{3}}\right),
$$

where $t \in[0,1]$. Thus for sufficiently large $l$ we know that $Y\left(1 ;\left(l+\frac{1}{2}\right)^{2} \pi^{2} ; t\right)$ is invertible for any $t \in[0,1]$, then $\operatorname{det} Y\left(1 ;\left(l+\frac{1}{2}\right)^{2} \pi^{2} ; t\right) \neq 0$ for all $t \in[0,1]$. Thus we can define the following integral

$$
M_{l}(t)=\frac{1}{2 \pi i} \oint_{|\lambda|=\left(l+\frac{1}{2}\right)^{2} \pi^{2}} \frac{\frac{\partial}{\partial \lambda} \operatorname{det} Y(1 ; \lambda ; t)}{\operatorname{det} Y(1 ; \lambda ; t)} d \lambda .
$$


According to (4), we have that

$$
Y(1 ; \lambda ; t)=\frac{\sin \sqrt{\lambda}}{\sqrt{\lambda}} I_{m}-\frac{t \cos \sqrt{\lambda}}{2 \lambda} \int_{0}^{1} Q(t) d t+t O\left(\frac{\exp \|Q\|_{\infty} \mid \Im \sqrt{\lambda \mid}}{|\sqrt{\lambda}|^{\frac{3}{2}}}\right) .
$$

Then the family $\{\operatorname{det} Y(1 ; \lambda ; t)\}_{t \in[0,1]}$ is a continuous family with parameter $t$. On the other hand, we know that $\frac{\partial}{\partial \lambda} \operatorname{det} Y(1 ; \lambda ; t)$ are sum of terms with multiplication of entries of $Y(1 ; \lambda ; t)$ and $\frac{\partial}{\partial \lambda} Y(1 ; \lambda ; t)$. Denote $Y_{\lambda}(x ; \lambda ; t)=\frac{\partial}{\partial \lambda} Y(x ; \lambda ; t)$. Then $Y_{\lambda}(1 ; \lambda ; t)$ satisfies the following equation:

$$
\left\{\begin{array}{l}
Y_{\lambda}^{\prime \prime}(x ; \lambda ; t)+\left(\lambda I_{m}-t Q(x)\right) Y_{\lambda}(x ; \lambda ; t)=Q(x) Y(x ; \lambda ; t), \\
Y_{\lambda}(0 ; \lambda ; t)=Y_{\lambda}^{\prime}(0 ; \lambda ; t)=0_{m} .
\end{array}\right.
$$

According to the continuity of $Y(x ; \lambda ; t)$ on $t$ variable, we know that $\left\{Y_{\lambda}(1 ; \lambda ; t)\right\}_{t \in[0,1]}$ is a continuous family with parameter $t$. Then $\left\{\frac{\partial}{\partial \lambda} \operatorname{det} Y(1 ; \lambda ; t)\right\}_{t \in[0,1]}$ is also a continuous family with parameter $t$. Thus $M_{l}(t)$ is a continuous function on $[0,1]$ with positive integer value, this shows that $M_{l}(t)$ is a constant function, $M_{l}(t) \equiv M_{l}(0)=l \mathrm{~m}$. From our argument of the distribution of the zeros of $Y(1 ; \lambda)$, we find that $L_{Q}$ has exactly $l m$ Dirichlet eigenvalues inside $\left\{|\lambda|=\left(l+\frac{1}{2}\right)^{2} \pi^{2}\right\}$. The above argument implies the following corollary:

Corollary 3. For $Q(x) \in C\left([0,1] ; \mathscr{L}\left(\mathbb{R}^{m}\right)\right), Q(x)=Q(x)^{*}$ for all $x \in[0,1]$. Let $\lambda_{n}$ be the $n$-th Dirichlet eigenvalue of $L_{Q}$, then

$$
\lim _{l \rightarrow \infty} \sum_{i=1}^{m}\left(\lambda_{l m+i}-(l+1)^{2} \pi^{2}\right)=\operatorname{trace}\left\{\int_{0}^{1} Q(t) d t\right\} .
$$

Proof. In the previous argument we find that there are $l \mathrm{~m}$ Dirichlet eigenvalues of $L_{Q}$ which are contained in $\left\{\lambda \in \mathbb{C}:|\lambda| \leqslant\left(l+\frac{1}{2}\right)^{2} \pi^{2}\right\}$. According to the identities (5) and (11), we find that

$$
\lambda_{l m+i}=(l+1)^{2} \pi^{2}+q_{i}+O\left(\frac{1}{l}\right)
$$

for $i=1,2, \cdots, m$. Thus

$$
\sum_{i=1}^{m}\left(\lambda_{l m+i}-(l+1)^{2} \pi^{2}\right)=\sum_{i=1}^{m} q_{i}+O\left(\frac{1}{l}\right) .
$$

Then our assertion holds.

From the previous argument for the multiplicity and the distribution of the Dirichlet eigenvalues of $L_{Q}$, we obtain the following theorem.

Theorem 4. Suppose that $Q(x) \in C\left([0,1] ; \mathscr{L}\left(\mathbb{R}^{m}\right)\right)$, and $Q(x)=Q(x)^{*}$ for all $x \in[0,1]$. Then all Dirichlet eigenvalues of $L_{Q}$ are of multiplicity $m$, if and only if there exists a bounded sequence $\left\{\tau_{n}\right\}_{n=1}^{\infty}$, such that

$$
\sigma_{D}\left(L_{Q}\right)=\left\{n^{2} \pi^{2}+\tau_{n} \mid n \in \mathbb{N}\right\} .
$$

Furthermore, $\left\{\tau_{n}\right\}_{n=1}^{\infty}$ is a convergent sequence, and

$$
\int_{0}^{1} Q(t) d t=\left\{\lim _{n \rightarrow \infty} \tau_{n}\right\} I_{m} .
$$


Proof. Suppose that $m_{g}\left(\lambda_{*} ; Y(1 ; \lambda)\right)=m$ for all $\lambda_{*} \in \sigma_{D}\left(L_{Q}\right)$. According to the identities (5) and (11), we find that

$$
\lambda_{l m+1}=\lambda_{l m+2}=\cdots=\lambda_{l m+m} .
$$

But $\lambda_{l m+i}=(l+1)^{2} \pi^{2}+q_{i}+O\left(\frac{1}{l}\right)$, thus $q_{1}=q_{2}=\cdots=q_{m}$ and $\tau_{l}=q_{1}+O\left(\frac{1}{l}\right)$. This implies that $\left\{\tau_{n}\right\}_{n=1}^{\infty}$ is a convergent sequence which converges to $q_{1}$, and

$$
\int_{0}^{1} Q(t) d t=q_{1} I_{m}
$$

Conversely, assume that there exists a bounded sequence $\left\{\tau_{n}\right\}_{n=1}^{\infty}$, such that

$$
\sigma_{D}\left(L_{Q}\right)=\left\{n^{2} \pi^{2}+\tau_{n} \mid n \in \mathbb{N}\right\}
$$

In the identity (24) we find that if $n \geqslant M_{Q}$, then $m_{g}\left(n^{2} \pi^{2}+\tau_{n} ; Y(1 ; \lambda)\right)=m$. Denote $\Gamma_{l}=\{\lambda \in$ $\left.\mathbb{C}:|\lambda|=\left(l+\frac{1}{2}\right)^{2} \pi^{2}\right\}$, the previous argument implies that $m_{a}\left(\Gamma_{l} ; Y(1 ; \lambda)\right)=\operatorname{lm}=m_{g}\left(\Gamma_{l} ; Y(1 ; \lambda)\right)$ for sufficient large $l$. From the distribution of $\sigma_{D}\left(L_{Q}\right)$, we find that there only $l$ eigenvalues inside $\Gamma_{l}$. Thus each eigenvalue inside $\Gamma_{l}$ is of multiplicity $m$. This implies that all Dirichlet eigenvalues of $L_{Q}$ are of multiplicity $m$.

Remark. Denote the $n$-th Neumann eigenvalue of $L_{Q}$ as $\mu_{n}$, then applying the argument similar to those argument in the proof of Corollary 3. and Theorem 4 , we have that

$$
\mu_{l m+i}=l^{2} \pi^{2}+q_{i}+o\left(\frac{1}{l}\right) .
$$

Furthermore, we also get that all Neumann eigenvalue of $L_{Q}$ are of multiplicity $m$, if and only if

$$
\sigma_{N}\left(L_{Q}\right)=\left\{n^{2} \pi^{2}+\epsilon_{n} \mid n \in \mathbb{Z}_{+}\right\},
$$

where $\left\{\epsilon_{n}\right\}_{n=0}^{\infty}$ is a bounded sequence. Applying these result, we can simplify the vectorial V.A. Ambarzumyan theorem (see [3]) as the followings:

Corollary 5. Let $Q(x)$ be a continuous $m \times m$ selfadjoint matrix-valued function, then $\sigma_{N}\left(L_{Q}\right)=$ $\left\{n^{2} \pi^{2} \mid n \in \mathbb{Z}\right\}$, if and only if $Q(x)=0_{m}$ for all $x \in[0,1]$.

From [Sh2] we know that if $Q(x) \in C\left([0,1] ; \mathscr{L}\left(\mathbb{R}^{m}\right)\right)$, and $Q(x)=Q(x)^{*}, Q(x)=Q(1-x)$ for all $x \in[0,1]$, and all eigenvalues of the equation (1) are of multiplicity $m$, then $Q(x)=q(x) I_{m}$, where $q(x)$ is a scalar continuous function defined in $[0,1]$, and $q(x)=q(1-x)$ for all $x \in[0,1]$. According to Theorem 4, we obtain the following result which is an extension of the previous inverse spectral theorem that we just mentioned. 
Corollary 6. Suppose that $Q(x) \in C\left([0,1] ; \mathscr{L}\left(\mathbb{R}^{m}\right)\right)$, and $Q(x)=Q(x)^{*}, Q(x)=Q(1-x)$ for all $x \in[0,1]$. Then the Dirichlet spectrum $\sigma_{D}\left(L_{Q}\right)$ of $L_{Q}$ is of the form

$$
\sigma_{D}\left(L_{Q}\right)=\left\{n^{2} \pi^{2}+\tau_{n} \mid n \in \mathbb{N}\right\}
$$

where $\left\{\tau_{n}\right\}_{n=1}^{\infty}$ is a bounded sequence, if and only if $Q(x)=q(x) I_{m}$ where $q(x)$ is a scalar continuous function defined in $[0,1]$, and $q(x)=q(1-x)$ for all $x \in[0,1]$.

\section{References}

[1] V. A. Ambarzumyan, Über eine Frage der Eigenwerttheorie, Z. Phys. 53(1929), 690-695.

[2] L. Bers, On Trace Formula, Lecture Notes in Mathematics Vol 925. The Riemann Problem, Springer-Verlag, 1980.

[3] H.-H. Chern and C.-L. Shen, On the n-dimensional Ambarzumyan's theorem, Inverse Problems, 13(1997), 15-18.

[4] I. Gohberg and S. Gohberg, Basic Operator Theory, (Basel: Birkhäuser), 1981.

[5] I. Gohberg, S. Gohberg and M. A. Kaashoek, Classes of Linear Operator vol.1 Birkhäuser, Basel, 1990.

[6] J. Pöschel and E. Trubowitz, Inverse Spectral Theory, Academic, New York, 1987.

[7] C.-L. Shen and C.-T. Shieh, Two inverse eigenvalue problems for vectorial Sturm-Liouville equations, Inverse Problems, 14 (1998), 1331-1343

[8] C-L. Shen, Some inverse spectral problems for vectorial Sturm-Liouville equations, Inverse Problems, 17(2001), 1253-1294

Department of Mathematics, National Tsing Hua University, Hsinchu, Taiwan, Republic of China.

E-mail: d907205@alumni.nthu.edu.tw 